\title{
Introduction of Durable Hydrophilicity on Nylon12 by Plasma Treatment
}

\author{
Masayuki Kuzuya,* Takashi Sawa, Tomoyuki Yamashiro, Shin-ichi Kondo and Osamu Takai ${ }^{1}$ \\ Laboratory of Pharmaceutical physical Chemistry, Gifu Pharmaceutical University, \\ 5-6-1 Mitahora-Higashi, Gifu 502-8585, Japan \\ kuzuya@gifu-pu.ac.jp \\ ${ }^{l}$ Graduate School of Engineering, Nagoya University, Furo-cho, Chikusa-ku \\ Nagoya 464-8604, Japan \\ takai@otakai.numse.nagoya-u.ac.jp
}

Key words: plasma treatment, surface hydrophilicity, Nylon12, maleic anhydridemethylvinylethel copolymer (GANTREZ), ESR, contact angle.

Considerable interest has focused on plasma treatment of hydrophobic polymer to enhance the surface wettability. The surface wettability of a polymer surface is an important characteristic which relates to the biocompatibility for biomaterials.") One of the advantages of plasma treatment is the fact that it is surface-limited so that only the surface properties are changed without affecting the bulk properties. It is known, however, that the surface wettability of many linear polymers introduced by nonpolymerforming plasma treatment using inert gas decays with time after treatment. This is a disadvantageous property of plasma treatment for practical use. The mechanism by which the surface wettability decays with time has been ascribed to several reasons: for plasma-induced crosslinkable linear polymers, the overturn and/or migration of hydrophilic functional groups into the bulk phase so that the hydrophobic moieties were reoriented towards the air interface, ${ }^{2)}$ and for plasma-induced degradable linear polymers, detachment of the hydrophilic group in lowermolecular weight species on the plasma-irradiated polymer surface. ${ }^{3)}$ Thus, the decay of wettability on the plasma-treated polymer surfaces is an intrinsic nature of plasma treatment of linear polymers due to the nature of reactivity of plasmainduced surface radicals, and it would be difficult to remove such a property by selecting the plasma operational conditions.
We have recently reported a novel method to introduce a durable wettability and minimize its decay with time on the poly(ethylenenaphthalate) (PEN) surface by a plasma-induced crosslink reaction to immobilize the hydrophilic carboxyl groups on the PEN surface derived from maleic anhydride-methylvinylether copolymer, commercially known as a GANTREZ. ${ }^{4)}$ PEN has been selected for an initial study of various polymers because PEN is one of the crosslinkable aromtaic containing polymers, and swells in a organic solvent such as tetrahydrofuran (THF).

We have also reported one example of a practical application for fabrication of highly fictionalized biomaterials of polyurethane-made catheter surface using the present method, demonstrating that the polyurethane-made cathether treated according to the present method under selected plasma operational conditions is of much higher durability of the surface lubricity. ${ }^{4 C)}$

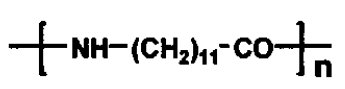

Nylon12

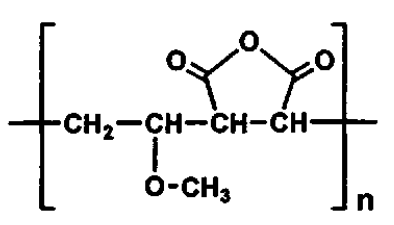

GANTREZ
Fig.1 Structures of Nylon 12 and GANTREZ. 
In this communication, we report a further extended work to introduce a durable wettability on the Nylon 12 surface according to the method as described above. Nylon 12 has been selected for the present study, since Nylon 12 is widely used as biomedical materials. The structural formula of Nylon 12 is shown in Fig.1.

In order to understand an Ar plasma-induced surface reaction of Nylon12, we have first examined the nature of Ar plasma-induced radicals of Nylon 12 based on the study of the electron spin resonance(ESR)spectra. The progressive changes of room temperature ESR spectra of plasmairradiated Nylon 12 powder with various plasma durations are shown in Fig. 2. Although the detailed study of ESR spectral analyses of Nylon 12 will be reported elsewhere, it was found that the simulated spectra are most characterized by the presence of three component spectra and are obtained from admixtures of all the component spectra with differing ratios. Figure 3 shows representative component spectra of the simulated spectra: a sextet spectrum(I) and a septet spectrum(II), assigned to the mid-chain alkyl radical and allylic radical, respectively, and a broad singlet spectrum(III) assigned to dangling bond sites (DBS) at the surface cross-linked network as in the case of other polymers.

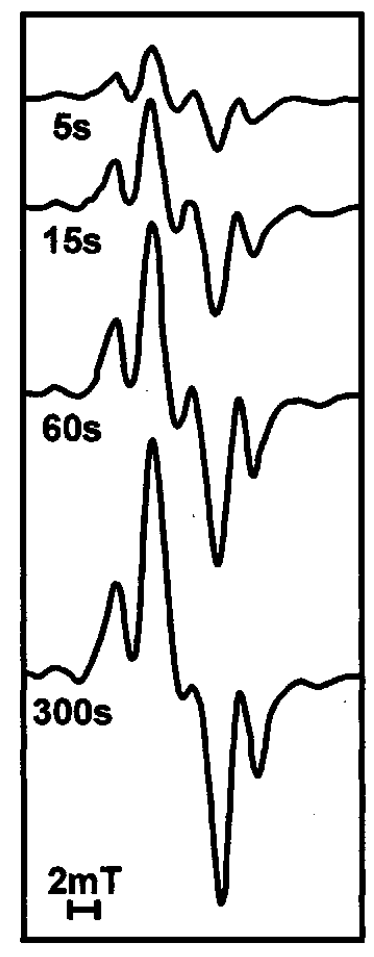

Fig. 2 Progressive changes in observed ESR spectra of plasma-irradiated Nylon 12.
The present result clearly indicated that a greater part of Nylon12 radicals including DBS were produced at the alkyl moiety of Nylon 12, and such component mid-chain radicals, I and II, have a great tendency to undergo the radical recombination reactions, based on the earlier ESR studies of polyethylene. ${ }^{5)}$ Thus, Nylon 12 can be considered to be a crosslinkable polymer as an effect of $\mathrm{Ar}$ plasma irradiation.

Figure 4 illustrates the method for the preparation of Nylon 12 with a durable surface wettability. A polymer precursor, a GANTREZ AN 139, was first deposited on a Nylon 12 polymer, and then the excess GANTREZ was removed from the surface with THF. The Nylon 12 thus treated was submitted to $\mathrm{Ar}$ plasma-irradiation to immobilize the GANTREZ soaked in the Nylon 12 by the surface cross-link reaction. Plasma state was sustained by inductively coupled plasma at $13.56 \mathrm{MHz}$ in $\mathrm{Ar}$ ( 0.5 Torr) for a prescribed period of time. Then, the hydrolysis of maleic anhydride linkage in GANTREZ was conducted by immersing the Nylon 12 in $0.1 \mathrm{M} \mathrm{NaOH}$ aqueous solution at $40^{\circ} \mathrm{C}$ to generate carboxyl groups and dried.

To evaluate the efficiency of the present method with the long term stability of acquired wettability of the Nylon12 surface, the water contact angle measurement of Ar plasma-irradiated Nylon12 film containing GANTREZ soaked was carried out by a drop-on-plate method using a goniometer-type contact angle meter, on its comparison with those of $\mathrm{O}_{2}$ plasma-irradiated film and non-plasmairradiated film containing GANTREZ. Figure 5 shows the progressive changes of the water contact

Alkyl radical
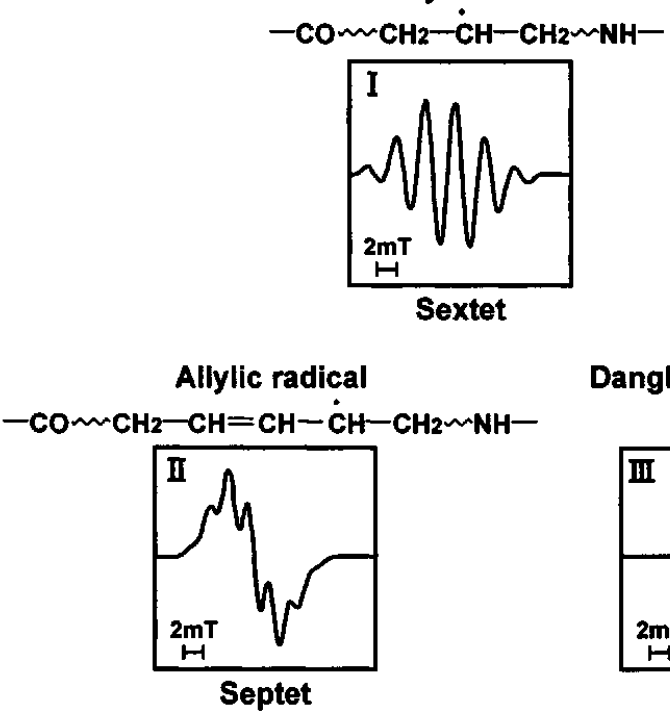

Dangling-bond sites (DBS)

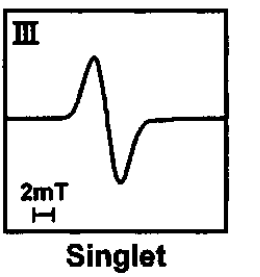

Fig. 3 Component spectra( I - III) for the simulated ESR spectra of plasma-irradiated Nylon 12. 


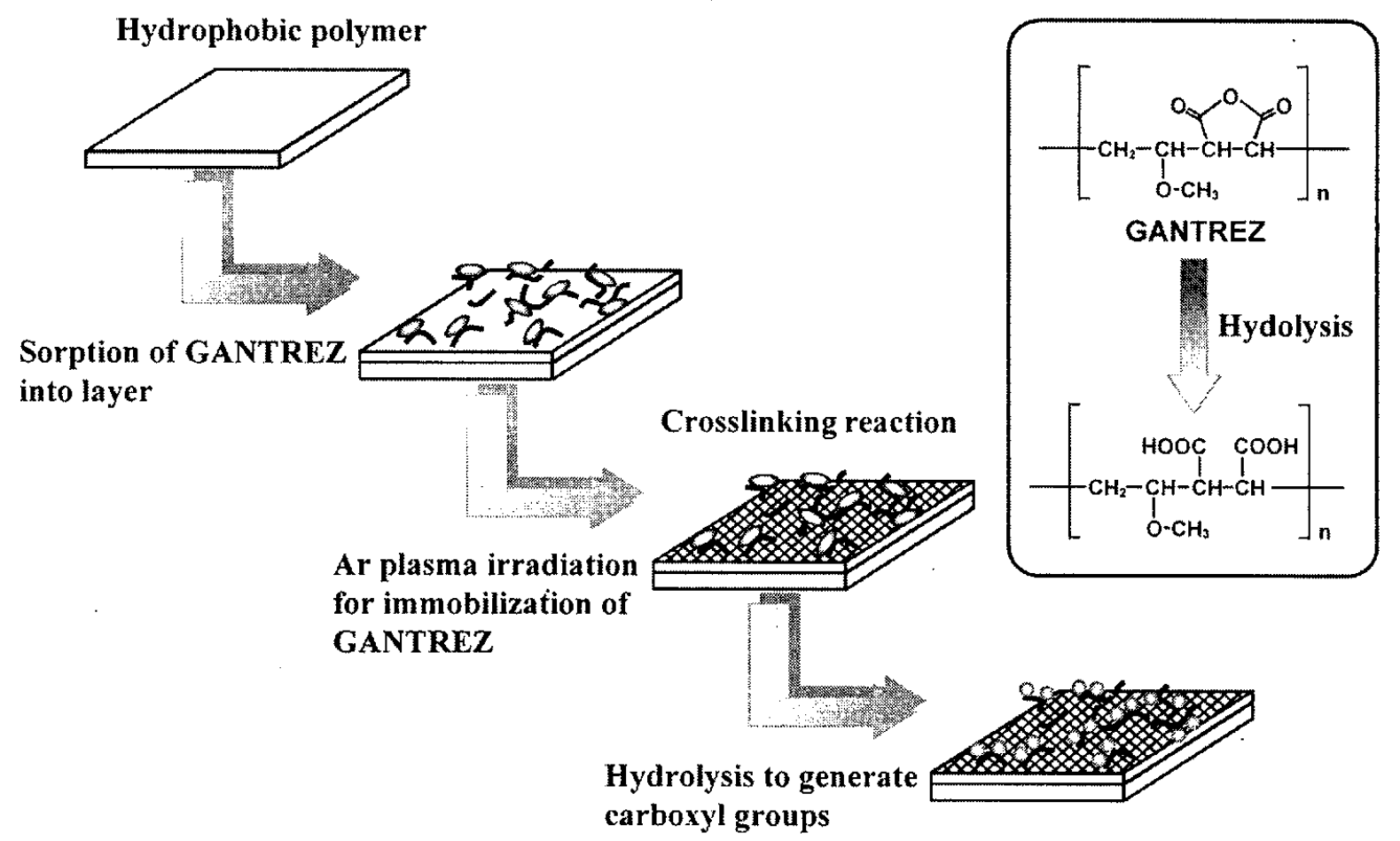

Fig. 4 Schematic illustration for fabrication of durable hydrophilicity onto polymer surface.

angles of Nylon12 film surfaces plasma-irradiated for $3 \mathrm{~min}$, when the sample was kept in water for prescribed period of time, and then dried. As is apparent from Fig. 5 that the contact angle of the Nylon 12 surface $\mathrm{O}_{2}$-plasma-irradiated for $3 \mathrm{~min}$ was largely decreased, but thereafter gradually increases as in the case of many other polymers. Non-plasmairradiated Nylon12 film treated with GANTREZ also showed the low contact angle at the beginning (immediately after plasma-irradiation) to such an extent as that of $\mathrm{O}_{2}$ plasma-irradiated Nylon 12 film due to the presence of hydrolyzates of GANTREZ at the Nylon 12 surface, but thereafter gradually increases and then becomes constant about $2 \mathrm{~h}$ later at the level around $55^{\circ}$, apparently due to the detachment of hydrolyzates of GANTREZ from Nylon 12 surface caused by the simple adsorption on the surface.

The water contact angle of Ar plasma-irradiated Nylon 12 film containing GANTREZ was similarly measured periodically. It is seen that the value of the contact angles of plasma-irradiated Nylon 12 film treated with GANTREZ was also largely decreased, but remained nearly constant at the level of initially acquired wettability for a long period of time. We believe the result would have stemed from an immobilization of hydrolyzate of GANTREZ by plasma-induced crosslinked surface of Nylon 12 .

We note here that the Nylon 12 film surface simply Ar plasma irradiated did not show the much difference in the value of the contact angle from that of a virgin Nylon 12 film. In order to find out the optimal plasma operational conditions for the long term stability of acquired wettability of the

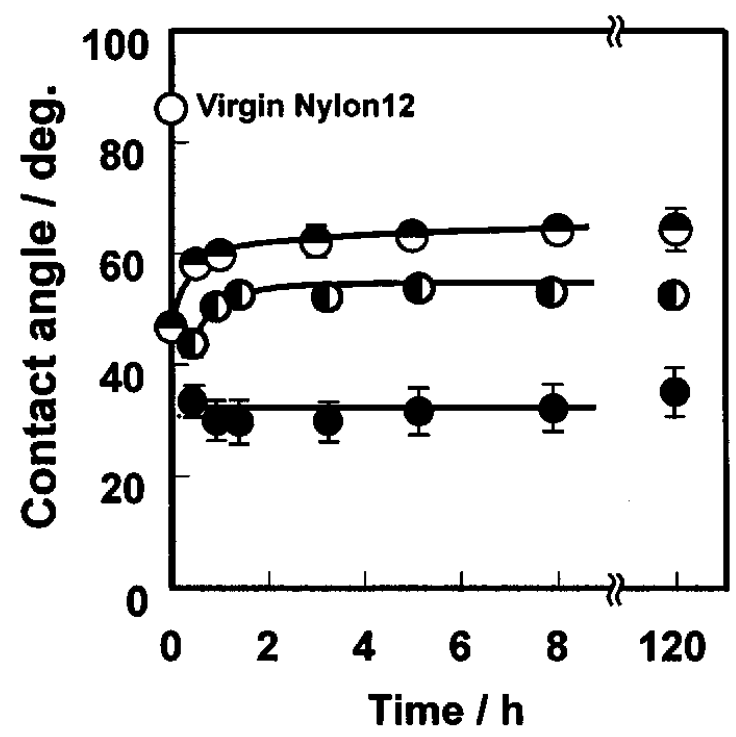

Fig. 5 Progressive changes of water contact angle of Nylon 12 film.

Plasma conditions : $40 \mathrm{~W}, 3 \mathrm{~min}, 0.5$ torr.

( $O$ ) $\mathrm{O}_{2}$ plasma-irradiated Nylon12 film; ( D ) non-plasma-irradiated Nylon12 film treated with GANTREZ; ( ) plasma-irradiated Nylon12 film treated with GANTREZ. 
Nylon12 surface, we have also examined the plasma irradiation on GANTREZ-treated Nylon12 film surface with various duration at output power of $40 \mathrm{~W}$ and $60 \mathrm{~W}$, and measured the contact angles of the resulted Nylon 12 surfaces after standing for $120 \mathrm{~h}$ in water.

Figure 6 shows the effect of plasma operational conditions on the water contact angles of GANTREZ-treated Nylon12 film surface. It is seen from Fig. 6 that the value of the water contact angle gradually decreased as the duration of plasma irradiation increased, but the value increased with prolongation of plasma irradiation, showing the minimum at about 90 s duration at $60 \mathrm{~W}$ and at about $150-180$ s duration at $40 \mathrm{~W}$, respectively. Thus, the result clearly demonstrated that it is also important to select the plasma operational conditions in order to introduce the surface wettability and the durability of acquired wettability into the Nylon 12 film surface treated according to the present method.

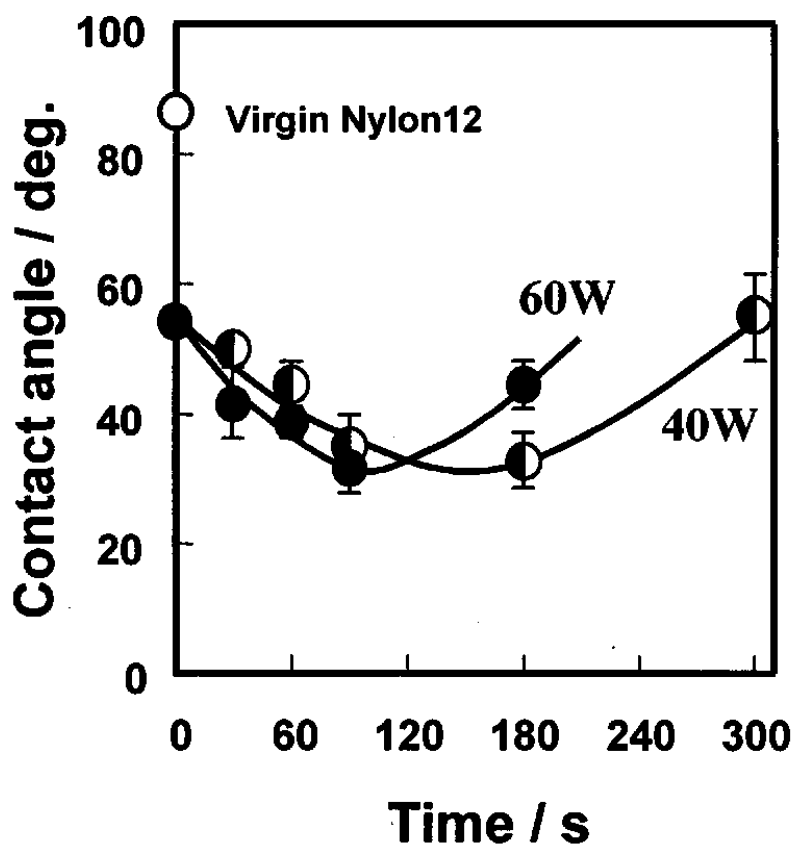

Fig. 6 Effect of plasma duration on water contact angle of plasma-irradiated Nylon12 film treated with GANTREZ after standing for $120 \mathrm{~h}$ in water.

Plasma conditions : 0.5 torr.

\section{Acknowledgement}

This work was supported in part by a Scientific Research Grant from the Research Project "Biomimetic Materials Processing"(No.JSPSRFTF 99R13101), Research for the Future(RFTF)
Program, Japan Society for the Promotion of Science, which is gratefuly acknowledged.

\section{References}

1. a) M. Hudis, Techniques and Applications of chemistry, J. R. Hollahan and A. T. Bell (eds.), John Wiley, New York (1974). b) H. Yasuda, Plasma Polymerization, Academic Press, Inc., New York (1985) c) K. Kamiya, Y. Yanagihara, T. Takai, and M. Kuzuya, Chem. Pharm. Bull. 42, (1996) 1896. d) B. D. Ratner, J. Photopolym. Sci. Technol. (Review) 8 ,

2. a) T. Hirotsu, and S. Onishi, J. Adhesion, 11 (1995) 481. See references cited therein. (1980) 57. b) H. Yasuda, and A.K. Sharma, J. Polym. Sci., Polym. Phys Ed., 19 (1981) 1285. c) Y. Ikada, K. Matunaga, and M. Suzuki, Nippon Kagaku Kaishi, (1985) 1079. d) Y. Taru and K. Takaoka Koubunshi Ronbunshu, 43, (1986) 361. e) M Morra, E. Occhiello, and F. Garbassi, J. Colloid Interface. Sci. 132, (1989) 504. f) M. Morra, E. Occhiello, R. Morra, F. Garbassi, P. Hymphrey, and D. Johnson, J. Colloid Interface. Sci. 137, (1990) 11. g) M. Morra, E. Occhiello, L. Gila, and F. Garbassi, j.Adhesion 33, 77(1990). h) E. Occhiello, M Morra, F. Garbassi, D. Johnson, and P. Hymphrey, Applied Surf. Sci. 47, (1991) 235. i) E. Occhiello, M Morra, P. Cinquina, and F. Garbassi, Polymer 33, (1993) 3007.

3. a) H. Schonhorn and F.W. Ryan, J. Polymer. Sci. A2 6, (1968) 231 . b) H. Schonhorn and F.W. Ryan, J. Polymer. Sci. A2 7, (1969) 105 . c) M. Kuzuya, A. Noguchi, Y. Tanaka, K. Sawada, D. T. Yang, Y. Yanagihara, and K. Kamiya, Proc. Jpn. Symp. Plasma. Chem., 2 (1989) 209.

4. a) M. Kuzuya and T. Yamashiro, J. Photopolym. Sci. Technol, 8, (1995) 381. b) M. Kuzuya, Y. Matsuno, T. Yamashiro, and M. Tuiki, Plasmas and Polymers, 2, (1997) $79 . \quad$ c) M. Kuzuya, Y. Matsuno, T. Yamashiro, and M. Tuiki, Plasmas and Polymers, 2 (1997) 133.

5. a) M. Kuzuya, J. Niwa, H. Ito, Macromolecules, 26, (1993) 1990 . b) M. Kuzuya, J. Niwa, T. Noguchi, Polymer J, 27 (1995) 251. c) M. Kuzuya, S. Kondo, M. Sugito, Polymer Preprints (ACS), 38, (1997) 1049. d) M. Kuzuya, M. Sugito, S. Kondo, J. Photopolym. Sci. Technol, 10, (1997) 135 . e) M. Kuzuya, T. Yamashiro, S. Kondo, Macromolecules, 31, (1998) 3225. f) M. Kuzuya, S. Kondo, M. Sugito, Macromolecules, 31, (1998) 3230 . 